\title{
A Multimodal Biometrics for Aadhaar Based Secured E-Voting System
}

\author{
K. Kanimozhi, K. Thangadurai
}

\begin{abstract}
False polling is still a significant issue in elections in the latest moments. In this job, an effort is made to fix this issue using current Aadhaar card database and electoral biometrics. This scheme authenticates electors by combining multimodal biometrics such as picture, eye, and palm printing, after which registration is verified by verifying the age that enables only qualified applicants to register. The time needed for authentication is decreased by using the corresponding Aadhaar amount and multimodal biometrics. This is authentication will be achieved by inspecting whether the registered Aadhaar amount and multimodal biometrics match or not without linking it with the entire biometric database to boost authentication pace. The polling of ballots will be performed automatically so that space is decreased and results can be announced in less moment. The improvisations strive to increase the system's safety, efficiency, efficiency, scalability. This suggested a safe internet e-voting system (EVS) that utilizes as its backend UIDAI or Aadhaar database. In this job, the entry pictures are originally preprocessed and the removal of features is achieved using Improved Gabor filters. Enhanced Support Vector Machine Algorithm (eSVM) is used to match and classify features development of this scheme will render the polling method more comfortable and can, therefore, contribute to enhanced turn-out. Using this multimodal biometric system for voting purposes, election rigging was easily avoided.
\end{abstract}

Index Terms: Aadhaar, UIDAI, enhanced Support Vector Machine Algorithm, median filtering

\section{INTRODUCTION}

India is one of the largest democracies in the world with a community of 1 billion. India has an electorate of around 700 million people. The voting theory started officially in the 18th decade and suggestions for the voting system have been created ever since. India is one of the biggest democracies in the world with a population of 1 billion. India has an electorate of around 700 million people. The voting theory started officially in the 18th decade and suggestions for the

Revised Manuscript Received on October 30, 2019.

* Correspondence Author

K.Kanimozhi*, Department of Computer Science, Bharathidasan University/ Government Arts College/, Karur, India.

Dr.K.Thangadurai, Department of Computer Science, Bharathidasan University/ Government Arts College/, Karur, India.

(C) The Authors. Published by Blue Eyes Intelligence Engineering and Sciences Publication (BEIESP). This is an open access article under the CC BY-NC-ND license (http://creativecommons.org/licenses/by-nc-nd/4.0/) voting system have been created ever since. This device is put in the center of the voting box and is supervised by greater authorities. Due to some illegal activities, the polling station is misused and people's vote to the right has been dismissed. This scarcely occurs in agricultural regions as well as in metropolitan towns because trained individuals are not keen on placing their ballots on applicants depicting their corresponding regions. Polling automation came into action to ensure 100 percent. But this automated system has only been endorsed in some developed countries since safety has not been assured to a big level. Our primary objective of the proposed system is to build a high-security, compatible voting system. The proposed system is mainly designed for our country. It has three phases first; the information of individuals over the era of 18 is obtained from the Aadhaar ticket database as it has become compulsory in the present situation. A fresh elector I'd with the necessary data will be automatically created and intimation will be supplied to people through their e-mail. When entering, the customer can indicate their I'd and password. The elector's multimodal biometrics (i.e., image, eye, and palm printing) are used as the main authentication instrument to ensure higher safety. Since the finger template of each human being is different, the elector can be easily authenticated. Face recognition, iris identification together with fingerprint-based authentication methods can be quite helpful in identifying people's identities. This research aims to incorporate face recognition, iris recognition and palm identification characteristics with Aadhaar based validation methods in present voting system hence the machine also helps to define and helpful in tracking electoral occurrences such as fraud, and other illegal activities neck gaiting in the case of elections. Scheme is embedded within multi-layer safety with a fingerprint authentication scheme that involves facial and iris recognition characteristics. For the application stage, the Voter must go to the authorized voting registration centers, and his biometric data such as picture, nose and thumb \#\#print with his electoral data must be recorded at the polling place. Once his register is complete, he can cast his votes from any of the authorized equipment. When polling is planned, individuals can go to the approved registered facilities. Since the electoral information is already accessible on the computer, the electorate and other information need not be executed. Only verification is enough to register the ballot. all suggest getting one of the biometric characteristics such as iris or face recognition or palm printing prior safety coating ,finger display the 2nd coating . 


\section{A Multimodal Biometrics for Aadhaar Based Secured E-Voting System}

If the thumb is left on the fingerprint scanner, the fingerprint information be requested and a model will be acquired and similar to the one already retained in the Aadhaar database. A data collected will be carried onto the control system for more validation and a unique fingerprint ID will be transferred once the binding has been completed.

The viewer acquired ID is driven by the current information already stored in the database. Once the data is found to match current database data, voters are allowed to enroll their vote. If not, an informative signal about the authentication error will be presented on the screen and the individual will not be refused entry to join his ballot. The main contribution of the work is as follows:

The first is the preprocessing unit, including noise removal, ROI identification, and normalization.

1. Noise elimination for the picture conducted with median filtering

2. Then normalize the volume of the picture and use the CLAHE algorithm to normalize the comparison.

3. Improved removal of the Gabor function for all three pictures

4. The feature vector is generated by combining all features and enhanced Support Vector Machine algorithm (eSVM) checks the authenticity of the voter.

\section{RELATED WORK}

Standard electoral systems, such as paper ballots, lever voting equipment, punched cards, GSM portable smart polling scheme, etc., have many drawbacks to address. Use biometric methods in [1] to achieve safer and more versatile real-time applications. Since this system has immediate monitoring, we can get the results faster and earlier. Some specifications were described in [2 ] depending on product design. Few are said to be the long lasting

battery control-unit, simpler and less costly components, etc., Then the control unit should be readily accessible also the device must readily handled. Voters need to have one of the switches, such as Aadhaar board, pan board, etc. There are two phases of tracking in [3]. First, the RFID tag is used which includes the validation information already provided in LPC 2148. The RFID tag includes a 2 significant parts of the transponder that are a small cabinet consisting of polling information. The transponder is surrounded by the antenna used to transfer energy to the transponder to pass collected information the RFID reader to check whether or not the elector adds to that particular polling booth. Second, the Fingerprint-scanner used for the RFID relates to that particular person. To attain greater safety, the author uses a distinctive technique called bio-hashing in [4]. To use this particular method, the hash code is created and use both hands ' hands and the hash password created has a 13-digit symbol that can be put as a barcode. To attain fingerprint value, the distinctive Minutia Points are calculated. The surveillance stage begins after the original stage above. Electors only have access to record efficiency games after inspecting for fingerprint. The voting phase begins after he has access to the EVM button to register their voting. The licensed repositories are discovered in EVM during the electoral processing \& enrollment stage, which are continually evaluated. The electronic voting device biometrically guaranteed [5] explains how to incorporate the EVM with the bio-metric
In [6],[7] the related concentrate is primarily on safety and security parts. These ideas prompted and provided the idea of using picture, eye and fingerprint authentication [8] to consider to use Raspberry Pi 3 to produce experimental kiosk prototype. We used raspberry pi 3 in our execution and in reality, functionalities such as image capture characteristics are embedded with efficiency in elections that register the picture of people [9],[10].The concept behind the multimodal verification scheme to improve safe monitoring polling or voting and to attain zero tolerance for fraud and other crimes. This paper therefore gives priority. The primary goal and purpose of the suggested Design is to have a safe, secure and intelligent polling machine with enjoyable, better thoughts for the comfort and comfort of the audience .Saleem Ulla Shariff et al. [13] suggested as "Face and Bio-Metric Based Assistance and Security System Using RFID and Arduino" a face and biometric authentication scheme.. Biometric methods enable a individual to be recognized immediately on the basis of physical or cognitive characteristics that relate to a certain individual. Each biometric characteristic has its boundaries and no biometric scheme is ideal so that a range of issues arise from biometric unimodal methods. Multimodal biometric devices are used to satisfy some of the listed inconveniences and constraints and to boost the amount of safety. This further section explains the main features of the multimodal biometric system: structure, fusion point, the methodology used to incorporate multiple verifiers and standardization techniques.

\section{PROPOSED METHODOLOGY}

The suggested scheme has two methods, the methods of enrollment and authentication. The enrollment method manages the entry method to enroll customers in the scheme; the enrollment mode yield will be deposited in the scheme database. The second method, which is the authentication method, addresses the method of reviewing the customers status by adding the users Aadhaar amount and multimodal biometrics and matching them with the databases; if the scheme discovered any comparable biometrics in the database, then the customer is entitled to register his ballot. The user's vote will be counted and stored in the database. The customer can inspect the election outcome by pressing the outcome key at the start of the polling cycle. The proposed system is illustrated in Fig.1.

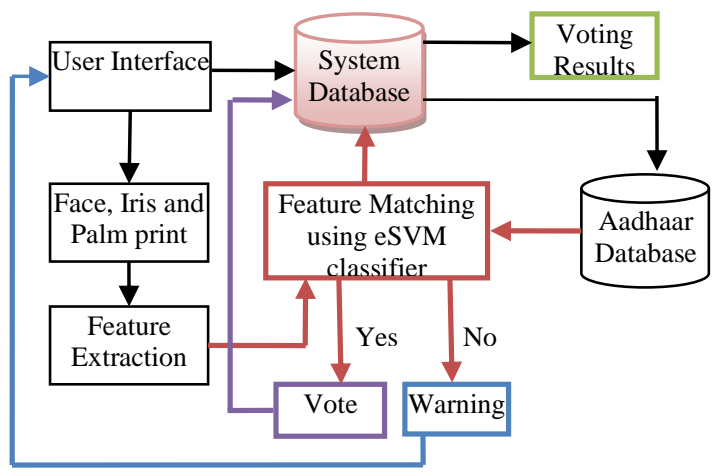

Fig.1. Multimodal Biometrics for Aadhaar Based Secure E-Voting System 


\section{A. IMAGE ACQUISITION}

The stage of Image Acquisition is the technique of acquiring images in different ways. The Aadhaar is used to record the fingerprint picture in this part head, iris, and palm print identity. The fingerprint image size will be $260 * 300$ pixels.

Face: Face recognition is a prevalent technique for individuals to recognize each other. The nose is a body's front from the chin to the forehead. Face recognition can be used in surveillance apps because the image is one of the few biometric characteristics that people can identify in a spectrum

Iris: Iris means behind the ear a ring-shaped cornea. The iris is very difficult to use after death because it is one of the flesh's first parts to disappear after death. The correct eye also differs from the remaining eye.

Palm Print: The human palm is the inner space between the thumbs and the wrist. The palm print area is much larger than the fingerprint region, and then it can extract more features than a fingerprint. The palm print is comparable to the fingerprint in hills and mountains, but the palm also has basic rows and wrinkles that can be obtained with a reduced definition microscope.

\section{B.IMAGE PROCESSING}

Normalization: First of all, there is a need to normalize each gray-level image to minimize differences between the original image contrasts. Let Img(x,y) the sample image's generic gray level; normalization is performed with respect to the maximum image. The outcome of normalization is the color enhancement of the color levels of the image.

ROI Extraction: This module converts pure biometric data obtained into a type appropriate for character processing. ROI is collected as a result of registration data. Depending on the characteristic, different algorithms are implemented to obtain the ROI.

ROI of Face: First, the system recognizes the face of the user in the instant of time $t$ within each frame $f r(t)$, using the well-known Viola and Jones method to obtain the initial region of interest of the face ROI. The ROI is standardized to a set magnitude and transformed into a pre-processed grayscale. At this point, the ROI is handled to remove noise, decrease air reliance and eliminate useless data. The ROI is divided into two sub-regions, ROI upper and ROI ground, respectively, to eliminate monitoring errors and reduce the processing duration of the system. Using both sub-regions enables for the elimination of insignificant data (e.g., teeth) and splits the removal method into two components (ears and mouth).

ROI of Iris: The Iris boundary is recognized in two stages. Image strength information is a separate border chart. Successively, the collection of border marks is entitled to vote to instantiate the contour of the parametric scores. The Canny algorithm is used to obtain the picture of the border. This method focuses on the magnitude limit of the image intensity gradient. Variables of image intensity are weighted to amplify certain color scores in order to incorporate directional modification. To recognize this boundary contour, the derivatives are weighted to be particular to vertical angles. Separate point voting method is introduced by the Canny operator to obtain separate spots along the boundary.

\section{ROI of Palm print}

Used to cultivate ROI palm painting following procedures. Two primary marks are selected from the set of nine primary points known. The first location is the valley mark in between the small finger and the ring finger, and the second place is valley peak between middle finger and the index finger These positions are considered towers of the anchor. The ROI spectrum is regarded as the range between the anchor columns and the triangle region's bottom angle is chosen as 30 pixels just below the first anchor stage. The outcomes of the preprocessing of the picture and the CLAHE phase are shown in fig.2.

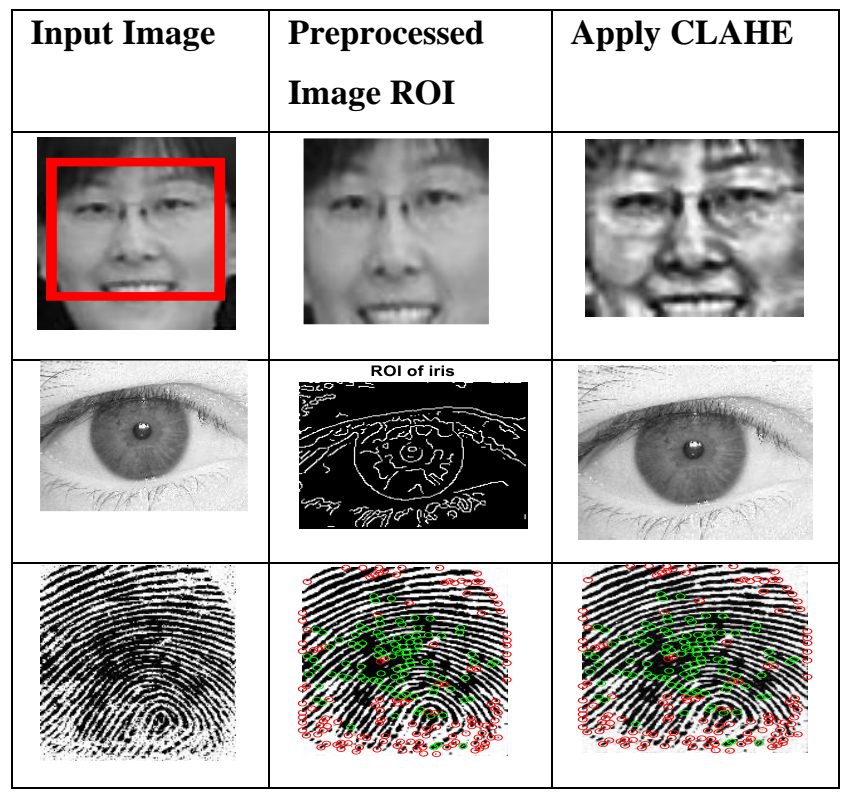

Fig.2.The results of Image preprocessing and CLAHE

\section{NOISE ELIMINATION USING MEDIAN FILTER}

De-noising algorithms could be easier if they include not only the noise but also the temporal features of the picture. Median Filter is a non-linear modeling technique that decreases the blurring of corners in which the concept is to substitute the present location in the picture with the neighborhood brightness average. Individual noise spikes do not affect the brightness index of the neighborhood, so median processing eliminates impulse noise quite well. In this experiment, the median filter was implemented and the average screened eye, iris, and palm picture was used as a nice performance picture for further improvement.

\section{D.IMAGE CONTRAST ENHANEMENT USING} CLAHE

Before extracting the feature, the image is enhanced by using Contrast Limited Adaptive Histogram Equalization (CLAHE), which is enhancement of Adaptive Histogram Equalization, as it overcomes a over-amplification for noise in homogeneous regions of an image, thus providing limited amplification. This move is essential to decrease noise and to solve poor brightness and illumination issues. It operates in small areas called rows and is used to softly combine neighboring areas with bilinear interpolation. 


\section{A Multimodal Biometrics for Aadhaar Based Secured E-Voting System}

\section{E.IMPROVED GABER FILTER BASED FEATURE}

\section{EXTRACTION}

Texture features play an important role in machine sight and model identification. Improved Gabor filter is used in this chapter to obtain texture characteristics that relate to multimodal biometrics such as head, iris, and palm printing. The time-frequency converted texture-based discrimination technique, which is accomplished in part oriented on enhanced Gabor filters.

Improved Gabor filters: The objective of edge identification for define a

feature's significant symmetry axis at a defined temporal scale. Local flow phase information can be obtained by converting the noise with a few quadrature filters (an uncommon filter and even filter). The use of two quadrature amplifiers allows the calculation of wave amplitude and stage at a defined temporal scale (temporal spectrum) location. The choice of quadrature filters is the enhanced

This can be achieved by building a filter bank using a amount of quadrature filters produced by rescaling the improved Gabor filter. Each scaling is intended to select specific frequencies of the data being evaluated Symmetry data is explored by discovering regions where the reaction of the even matrix dominates the reaction of the specific experiment being differentiated from its complete scores transition equation $(G)$ of an enhanced Gabor model in the noise domain is built as a result of two parts: a one-dimensional Log Gabor matrix that controls the scores to which the filter responds and a circular reversed linear Gaussian function that controls the selectivity of the filter's location.

$$
\mathbb{G}(\omega, \phi, \theta)=\exp \frac{\left(\log \left(\omega / \omega_{0}\right)^{2}\right.}{2\left(\log \left(\kappa / \omega_{0}\right)\right)} \times \exp \left(-\frac{\alpha((\phi, \theta))}{2 \sigma_{\alpha}^{2}}\right)
$$

Were $\omega$ is oscillation frequencies, $\alpha$ is the scale factor, and $\kappa$ is the number of filters.

Texture Edge Detection of Multimodal Biometric Images: The stages of fabric border identification are described below. For the Multimodal Biometric Images, the results of the ideal processing stages for each sequence are shown.

Step 1 (Filtering): The Gabor filter and wavelet filter are implemented in one position (along all the vertical rows of the picture) along with the collection of all Image I parallel lines. The output is supplied as a Gabor filter

$$
H_{k}\left(x_{c}, y\right)=I\left(x_{c}, y\right) * g_{k}
$$

Where $*$ displays the convolution operator, gk reflects the Gabor filter with the specified parameter $\mathrm{k}=(\mathrm{u}, \mathrm{l}), \mathrm{I}(\mathrm{xc}, \mathrm{y})$ indicates the $\mathrm{x} \mathrm{c}$ th row of picture I, and Hk indicates the kth filter reaction. The corresponding manufactured images for a set of four Gabor filters are $\mathrm{H} \mathrm{1,...,} \mathrm{H} \mathrm{4.Similarly,} \mathrm{using} \mathrm{the}$ two most frequently used DWT, Image I is handled in the same way. Let $\mathrm{H} \mathrm{5,} \mathrm{H} \mathrm{6,} \mathrm{H} 7$ and $\mathrm{H} 8$ indicate the answers to the filter. Where $\mathrm{H} 5$ and $\mathrm{H} 6$ show extensive decomposition of the filter sub-band level-1 and level-2 respectively, and $\mathrm{H}$ 7 and $\mathrm{H} 8$ imply extensive decomposition of the Gabor sub-band level-1 and level-2 respectively.

Step 2 (smoothing): Filtered pictures acquired in step 1 are blurred with asymmetric Gaussian filter.

$$
V_{i}(x, y)=H_{i}(x, y) * L(x, y)
$$

Where, if images are tested along concurrent vertical lines and if images are scanned along parallel lateral lines Vix, the transformed output of $\mathrm{I}$ th image is indicated where $\mathrm{I}=1, \ldots 8$. Step 3: Steps 1 and 2 are repeated to obtain manipulated images in the orthogonal direction. Let F I (x, y) show all tested photos where $\mathrm{I}=1, \ldots, 16$. Thus, a 16 -dimensional array $\mathrm{F}(\mathrm{x}, \mathrm{y})$ is obtained as: $\mathrm{F}(\mathrm{x}, \mathrm{y})=[\mathrm{F} 1(\mathrm{x}, \mathrm{y}), \ldots \mathrm{F} 16(\mathrm{x}, \mathrm{y})$

Step 4: Using the texture boundary classification, a one-dimensional feature graph $\{\mathrm{F}(\mathrm{x}, \mathrm{y})\}$ is generated over the vectors. For each pixel $(x, y)$, the scalar index $M(x, y)$ of the reference vector closest to $\{\mathrm{F}(\mathrm{x}, \mathrm{y})\}$ is assigned.

$M(x, y)=\arg \min _{\mathrm{i}}\left\|F(x, y)-w_{i}\right\|$ for $w_{i} \in \Gamma$

In this way, transform the vector image to a scalar image. Feature map $M$ is smoothed with asymmetric Gaussian

$$
E(x, y)=M(x, y) * L(x, y)
$$

Where, Lx, y indicates Gaussian filter and E indicates a smooth picture. The function chart (scalar picture) acquired from the pictures processed images is shown.

Step 5 (Canny edge detection): Canny's edge detection technique is added to the blurred function chart picture $\mathrm{E}$ acquired in the past phase to achieve the border graph.

Step 6 (edge linking): Using the comparable algorithm, the discontinuities in the border chart acquired using canny edge detection are related. The method measures are as follows:

(i) Find all parts c 1,c 2, ..., c b, ..., c B obtained with canny edge identification in the boundary graph. Where c b shows a segment of the connected edge and c B shows the total number of connected segments. Repeat phase (ii) from $\mathrm{b}=1, \ldots, \mathrm{B}$

(ii) (ii) Find the starting points of the related chapter c b. Where start lines are the nodes among their 8-Neighbors that comprise only one border pixel. Connect the beginning points of the $\mathrm{c} b$ the segment to the nearest boundary pixel (other than pixels in CB the part) or image boundary blocks

(iii) Final edge map is obtained.

Step 7 (Feature fusion): one of the easiest types of fusion of function type is to concatenate the extracted features. In this task, function point fusion is accomplished by enhancing the uniform function vectors obtained from the user's face and shoulder modalities and performing concatenated vector feature choice. Let $\mathrm{F} i=\{\mathrm{f} 1, \mathrm{f} 2, \ldots, \mathrm{fn}\}$, Ir $\mathrm{i}=$ \{particularly Ir 1 , especially Ir $2, \ldots$ The function matrix of the user's image modalities, Iris modalities, and crown mapping modalities is represented by the $n\}$ and $P i=\{P 1, P 2, \ldots, P n\}$. The purpose is to combine F I roll over I and PI feature set to produce a new feature vector called Fv I that would better represent the individual. The vector Fv I is generated by first normalizing the vectors F III and P I and then concatenating the resulting vectors of the feature. Then the selection of the feature is performed on the combined feature vector of $\mathrm{Fv}$ i.

Step 8 (Feature Selection): In addition, some of the function scores may be ' loud ' relative to others. The feature selection method includes choosing a minimum function array of length $k, k<(m+n)$, which increases ranking efficiency on a practice collection of function vectors. The choice of immediate forward moving [14] method is used to conduct feature selection on Fv I function scores. 


\section{F.AADHAAR BASED E-VOTING WITH eSVM}

The suggested scheme is a safe e-voting scheme that utilizes as its backend India's Unique Identification Authority (UIDAI) or Aadhaar repository. The scheme guarantees an individual's authentication by combining pictures; eye and thumb color and registration are verified by calculating the voting age, rendering the current polling machines useless by using eSVM.

The eSVM by modifying kernels: In this work, Traditional SVM efficiency is improved by altering the Gaussian kernel to increase spatial resolution by conformal boundary tracking to enhance identifying precision. It focuses on the geometric structure of Riemann triggered by the kernel function. This section proposes an enhanced multi-classification Support Vector Machine (eSVM) method. Kernels offer additional support vector machines with the capacity to link non-linearly separable data collections to a different realm where they are linearly separable. Mapping links greater information sizes involves costs. Fortunately, SVMs obviously need not position these large-dimensional vectors. The position registration information in the larger dimensional and show internal products. Varied kernel features give various mappings. In this chapter, the current Gaussian kernel needs to be changed according to the specific electoral system. This altered kernel gives more effectiveness relative to the original Gaussian kernel. The objective function model of ESVM is given in Fig.3

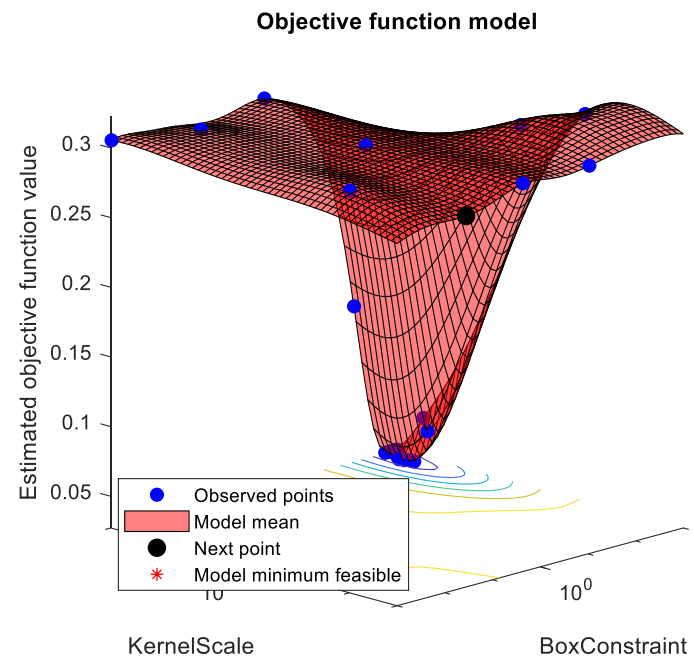

Fig.3.EVM with the objective function model

A nonlinear SVM converts every sample of admission room $\mathrm{R}$ to Fv Functionality space via non-linear transformation. The loc of the chart defines an embedding of $\mathrm{S}$ in $\mathrm{Fv}$ as a sub-manifold curve. Denotes $S$ measured sample in Fv space; small $\mathrm{dx}$ vector is measured and labeled as follows:

$$
\emptyset(d x)=\sum_{i} \frac{\partial}{\partial x^{(i)}} \emptyset(x) d x^{(i)}
$$

The squared length of $\emptyset(d x)$ is written as:

$$
|\emptyset(d x)|^{2}=\sum_{i, j}\left(\frac{\partial}{\partial x^{(i)}} \emptyset(x) \cdot \frac{\partial}{\partial x^{(j)}} \emptyset(x)\right) d x^{(i)} d x^{(j)}
$$

Where $\frac{\partial}{\partial x^{(i)}} \emptyset(x) \cdot \frac{\partial}{\partial x^{(j)}} \emptyset(x)=g_{i j}(x)$ is the Riemannian metric tensor and so the squared length of $\emptyset(d x)$ is rewritten as follows:

$$
|\emptyset(d x)|^{2}=\sum_{i, j} \mathcal{R}_{i j}(x) d x^{(i)} d x^{(j)}
$$

The column denoting the summation over the $\alpha$ coefficient of anywhere. The Positive-definite vector $\mathrm{R}$ ij ( $\mathrm{x}$ ) is caused in S and thus described as follows:

$$
\mathcal{R}_{i j}(x)=\frac{\partial}{\partial x^{(i)}} \varnothing(x) \cdot \frac{\partial}{\partial x^{(j)}} K\left(x, x_{i}\right)
$$

We can improve the variety or variations (D) in between class improve the effectiveness of the SVM. The metric tensor of Riemann rises around the border and decreases it around other samples. Kernel $\mathrm{K}$ can be changed in such a manner that $\mathrm{R}$ ij ( $\mathrm{x}$ ) is about the threshold as outlined below. Assuming the kernel can be modified as follows and can be called by factor $\mathrm{p}(\mathrm{x})$ as a conformal transformation of a kernel:

$$
\widetilde{K}\left(x, x_{i}\right)=p(x) p\left(x_{i}\right) K\left(x, x_{i}\right)
$$

The kernel function used in SVM is Gaussian Kernel,

$$
K\left(x, x_{i}\right)=\exp \left(-\frac{\left\|x-x_{i}\right\|^{2}}{\sigma^{2}}\right)
$$

Here, the parameter $\sigma$ is kernel width. It is proved that the corresponding Riemannian metric tensor is changed into:

$$
\mathcal{R}_{i j}(x)=\frac{1}{\sigma^{2}} \delta_{i j}
$$

After modifying the kernel Riemannian metric tensor is changed into:

$$
\tilde{\mathcal{R}}_{i j}(x)=p_{i}(x) p_{j}(x)+p^{2} \mathcal{R}_{i j}(x)
$$

To ensure that $p(x)$ has a large value around the support vector (SV), by the conformal transformation of the Gaussian kernel,

$$
p_{i}(x)=\frac{\partial p(x)}{\partial x_{i}}
$$

The quantity of $\mathrm{p} I(\mathrm{x})=0$ for highest $\mathrm{p}(\mathrm{x})$. To guarantee that $\mathrm{p}(\mathrm{x})$ has big numbers at aid vector locations, it can be built in a data-dependent manner such as:

$$
p(x)=\sum_{i \in S V} \alpha_{i} \exp \left(\frac{-\left\|x-x_{i}\right\|^{2}}{2 \mathfrak{f}^{2}}\right)
$$

Where $\mathrm{f}$ is a free parameter and where summation across all assists vectors. Since $\mathrm{p} \mathrm{I}(\mathrm{x})$ and $\mathrm{p}(\mathrm{x})$ are big when $\mathrm{x}$ near to supporting pillars and tiny when $\mathrm{x}$ is removed from SVs, the $\mathrm{R}$ ij (x) around energy items is reinforced when $\mathrm{x}$ is near to helping artifacts. As a consequence, the spatial resolution around the border is expanded and SVM's capacity to rate is enhanced. The suggested software method is described as follows: Step 1: Train SVM with the main Gaussian matrix $\mathrm{K}(\mathrm{x}, \mathrm{x} \mathrm{I}$ to collect SV data, then alter the Gaussian matrix K. Step2: Train the SVM with final Gaussian matrix $\tilde{K}\left(\mathrm{x}, \mathrm{x} \_\mathrm{i}\right.$ ) Step3: Use the above two measures as an iterative until the highest output is attained.

Authentication and Verification of the Voter Authentication: It is the technique of determining whether someone or something is actually who or what it is asserted to be. We make sure they have a appropriate Unique Identification (UID) number / Aadhaar code to authenticate an person. The quantity will first be confirmed in the local records of the database. If this is not found, the primary database will be scanned. That involves one-to-many matches. If the amount of the person is not discovered in the main database, he / she will, of course, prevent having a place in the electoral system. On the other hand, if the number is in the central database, that person's data will be cached to the local database. 


\section{A Multimodal Biometrics for Aadhaar Based Secured E-Voting System}

The person's multimodal biometrics such as face, iris, and palm print will be scanned on the client side and matched on the servers with data extracted from the local database using eSVM. In addition, Aadhaar data would be insufficient to produce a person's real identification as they can readily be faked, but using multimodal biometrics it is guaranteed that such fake entries are prohibited at the very start. In very situations where multimodal biometric identification does not work, the authentication of the voter is verified by his / her unique iris pattern.

Preventing Fraudulent Voting: In this, the first and primary role of securing suitable elections is performed by correctly authenticating each elector. Defining that each person who comes to parliament is unique is crucial; otherwise it violates the very notion of democracy. Any individual would be acting on behalf of others. Multimodal biometric bonding guarantees the scheme needs authentication. However, in an attempt to improve precision,

it is essential to maintain the fake rejection rate (FRR) and the fake authorization frequency (FAR) as small as feasible; practically near to null. Using this suggested model, this can be accomplished. To avoid the registration of underage people, the scheme calculates the lifespan of the person in the database documents from the place of conception. If the calculated era exceeds the permissible threshold, the individual may be enrolled and otherwise avoided. We implement voting caps in local databases to prevent voters from recording two or more successive times. Initially, this sign is put to true. After the poll has been done, his / her electoral cap will be set to true. This will stop the same individual from polling again. Signs are provisional and can be adjusted once the contest is over so that the elector can engage in the next election. Finally, the results from all the local databases are analyzed to obtain the initial biometrics of the applicant. This scheme therefore offers instant outcomes and avoids excessive use of resources and expenditure moment as it is an electronic scheme that utilizes digital data which has several advantages. Statistics can be produced from the information collected, for instance, how many individuals registered from a certain region, how many women registered, which gender bracket registered the most, the largest turnouts, differences from previous years, etc. All this was possible from traditional voting methods, not even from EVMs. It would provide important views on the election results and more enhance the scheme.

\section{RESULTS AND DISCUSSION}

This system uses the UIDAI or Aadhaar repository as its backend. By using this database, the data of the voter will be deposited on the personal computer. Multimodal biometric testing could also be an honorable option for e-voting applications where you can provide appropriate input and instruction to customers and where the scheme works in a regulated setting. The scheme guarantees an individual's authentication by adding Aadhaar amount and registration is verified by calculating the voter's era, thus rendering the current electoral machines useless. Performance is measurements depending on the spatial resemblance of the F-measure (SSIM) and precision of identification.

\section{A.SSIM PERFORMANCE COMPARISION}

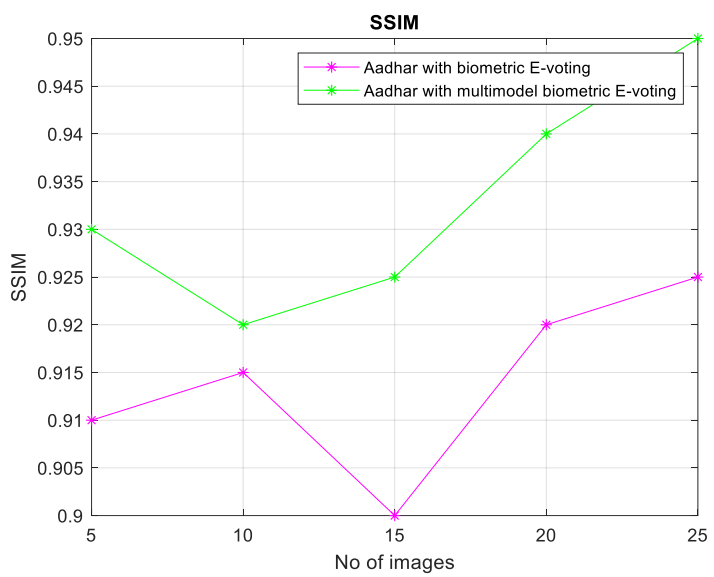

Fig.4. SSIM performance comparison in various compression techniques

Fig.4 shows the results of the SSIM comparison between the Exiting Aadhaar-based single biometric scheme and the proposed multimodal biometric scheme. From the assessment, the suggested method can attain high SSIM compared to present methods is an effective path to get the lung nodule at the high SSIM rate of 0.95 at the image size of 25 properly. Comparing the SSIM between current techniques at the same image size of 25 , giving a reduced price than the suggested technique. Through the results, it can be seen that the suggested work is much higher than the present method of an effective e-voting scheme.

\section{B. F-MEASURE PERFORMANCE COMPARISION}

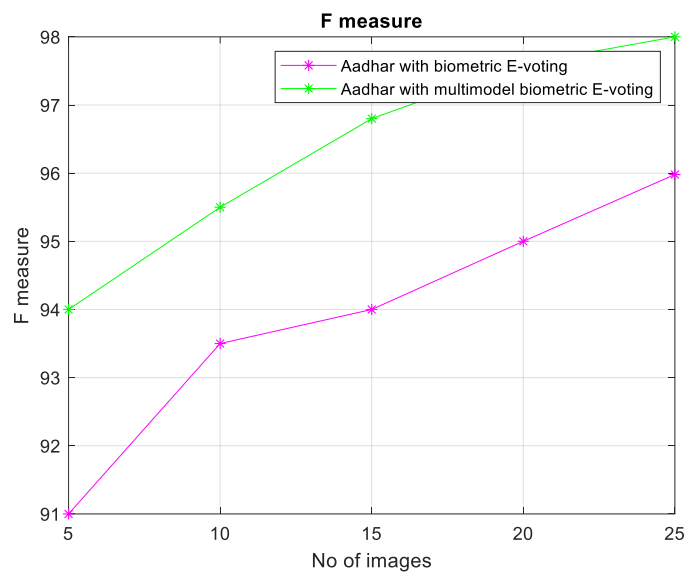

Fig.5. F-measure performance comparison in various compression techniques

Fig. 5 shows the results of the F-measure comparison between the Exiting Aadhaar-based single biometric scheme and the proposed multimodal biometric scheme. The technique suggested has large F-measure importance. It is well recognized from the outcomes that the suggested technique obtains an elevated F-measurement frequency showing the excellent vote tracking outcome. Because the suggested system is focused on great feature extraction and the idea centered on eSVM improves teaching effectiveness. Comparing the F-measure frequency between the present methods at the same image size of 25, providing an F-measure frequency of 95.98 that is 2.02 smaller than the suggested method. As a consequence, shows that the suggested job can provide stronger genuine electoral identification.

\section{C.DETECTION ACCURACY}




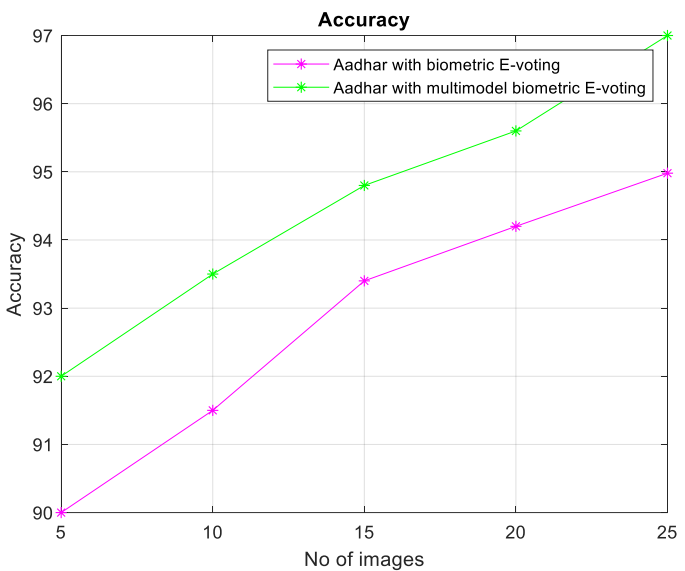

Fig.6.Detection Accuracy Vs No. of Nodes

Fig. 6 shows the blended accuracy of Exiting Aadhaar-based single biometric scheme and proposed multimodal biometric scheme under the number of images. It is observed that the monitoring accuracy of the suggested method is greater than that of the present scheme. From the graph,

it is observed that when the quantity of images increases, the average DA in the present method reduces. When the suggested method is implemented, the average monitoring accuracy of the suggested method and present strategy is 97 percent and 94.98 percent respectively. The cause is that; the eSVM will efficiently enhance the method of authenticated electoral identification.

\section{CONCLUSION}

The proposed voting system had many advantages over the traditional voting method. This system provides additional security by allowing voters to enroll once only by providing unique identification along with multimodal biometric data such as face, iris, and palm printing. This scheme prevents illegal polling and illegal behavior during the contest, which is the main problem in the tradition. The benefits of this scheme are financial tabulation, quicker production, enhanced accessibility, higher precision, and decreased probability of private and mechanical mistakes. A database composed of information such as era, people's biometrics should be changed every moment before voting. Information on set ballots can be sent to the elector through the communication system. Developing a set of metrics to recognize abnormal client practices by recognizing their physiological and cognitive features is a major issue in the future, and recognizing false clients with an effective classifier is a motivational element.

\section{REFERENCES}

1. Anandaraj , S,Anish R, Devakumar P.V "Secured Electronic Voting Machine using Biometric" IEEE Sponsored 2nd International Conference on Innovations in Information, Embedded and Communication systems (ICIIECS)2015.

2. Naik, Devendra Vijay. "Smart wireless authenticating voting machine." In 2015 International Conference on Communications and Signal Processing (ICCSP), pp. 0785-0788. IEEE, 2015.

3. B.Madan Mohan Reddy, D. SrihariRFID "Based Biometric Voting Machine Linked To Aadhaar For Safe And Secure Voting",International Journal of Science, Engineering and Technology Research (IJSETR) Volume 4, Issue 4, April 2015

4. Arooj, A. and Riaz, M., 2016, August. Electronic voting with biometric verification Offline and Hybrid EVMS solution. In 2016 Sixth International Conference on Innovative Computing Technology (INTECH) (pp. 332-337). IEEE.
. Abdur Rahman, "Biometrically secured electronic voting machine", (R10-HTC) DOI: 10.1109/R10- HTC.2017.8289010

6. J.C.Narayana Swamy, Dr. D Seshachalam, Saleem Ulla Shariff , "Smart RFID based Interactive Kiosk Cart using wireless sensor node". IEEE International Conference on Computational Systems and Information Technology for Sustainable Solution. CSITSS-2016, pp. $459-464$

7. Saleem Ulla Shariff, Maheboob Hussain, Mohammed, Farhaan Shariff. "Smart unusual event detection using low resolution camera for enhanced security", 2017 International Conference on Innovations in Information, Embedded and Communication Systems (ICIIECS), 2017

8. Saleem Ulla Shariff, C Amaranatha, Ravi Anand Jadhav, Dr. K Suresh Babu, Maheboob Hussain, "Face and Bio-Metric Based Attendance and Security System using RFID and Arduino", 4th National Conference on Networking Embedded and Wireless Systems NEWS-2016 International Journal of Electrical Electronics \& Computer Science Engineering Special Issue, pp. 84-89.

9. Nicole J. Goodman ; Jon H. Pammett, "The patchwork of internet voting in Canada", 2014 6th International Conference on Electronic Voting: Verifying the Vote (EVOTE), Pages: 1 - 6

10. Bhuvanapriya R., Rozil Banu S, Sivapriya P, Kalaiselvi V.K.G, "Smart voting”, 2017 2nd International Conference on Computing and Communications Technologies (ICCCT), Year: 2017, Pages: 143 147

11. K. Srinivasan, K. Pokumaran and G. Sainarayan, "Improved Background Subtraction Techniques for Security in Video Application", Anti-counterfeiting Security and Identification in Communication, pp. 114-117, 2009.

12. Sugandi, B., Hyoungseop Kim, Joo Kooi Tan, Ishikawa, "Tracking low resolution objects by metric preservation", in Computer Vision and Pattern Recognition (CVPR), 2011, pp. 1329-1336.

13. Saleem Ulla Shariff, C Amaranatha, Ravi Anand Jadhav, Dr. K Suresh Babu, Maheboob Hussain, "Face and Bio-Metric Based Attendance and Security System using RFID and Arduino", 4th National Conference on Networking Embedded and Wireless Systems NEWS-2016 International Journal of Electrical Electronics \& Computer Science Engineering Special Issue, pp. 84-89.

14. P. Pudil, J. Novovicova, and J. Kittler, "Floating search methods in feature selection," Pattern Recognition Letters 15, pp. 1119-1124, November 1994. 